\title{
An approach to implement interactive teaching in blended learning environments
}

\author{
Mohamed Sayed \\ Faculty of Computer Studies, Arab Open University (On Leave from Alexandria University, Faculty of Engineering) \\ E-mail:msayed@aou.edu.kw
}

Copyright $\odot 2015$ Mohamed Sayed. This is an open access article distributed under the Creative Commons Attribution License, which permits unrestricted use, distribution, and reproduction in any medium, provided the original work is properly cited.

\begin{abstract}
The use of electronic response systems in classrooms can be effective, especially when coupled with a method allowing a question to be broken into manageable interactive questions. This paper develops the use of electronic response systems as a part of leading assessment delivery and data collection solutions in a blended learning environment. The technology that is the subject of this research consists of thirty-button response cards that transmit student responses to a receiver connected to the instructor laptop. The electronic response systems are proven to enhance the effective instructions, increase retentions, engage participants, and immediately assess understanding.
\end{abstract}

Keywords: Blended Active Learning; Classrooms Technology; Computer-Marked Assessment; E-Instructions.

\section{Introduction}

According to Guthrie et al [5], students are mainly active learners. Encouraging active learning has long been considered as one of the main principles for good practice in higher education [2], [9], and [10]. A technology that offers one approach to implement active learning is clickers, or more formally Student Response Systems (SRS). Clickers are response-pads that allow students to select anonymously an answer to multiple-choice, true/false, and yes/no questions displayed by the lecturer. Students' responses are instantly transmitted to a receiver connected to the classroom computer, and the results are graphically displayed in real-time. Using SRS has much to offer in terms of amending the student learning experience. It is worth mentioning that the term objective questions, practiced to describe multiple choice questions in particular, reflect the fact that the early use of multiple-choice came from a desire to make assessment more objective. However, multiple-choice testing as an educational tool gained in popularity during the $20^{\text {th }}$ century as researchers became more being aware of the limitations of essays, see [7], [13].

Moreover, several benefits of using student response systems have been reported in technical literature. While most studies agree that SRS improved student in-class engagement; the findings related to the impact of SRS on exam scores were mixed. Two studies [4], [5] reported a significant increase of student participation where student response systems are used, partly because of anonymity, ease of use, and the possibility to see others' responses. Another study in the nursing literature [12] showed that students demonstrated positive attitude and a perceived benefit with the use of the SRS. The same study reported little improvement in exam scores. These results were supported by a study in an introductory psychology class $(n=1290)$ [8], where the findings revealed a small positive effect on the exam scores.

On the other hand, in a study with 1221 students in chemistry courses, Hall et al [6] confirmed the improved students' engagement and increased learning. However, unlike above studies, Hall et al reported a significant improvement in exam scores with respect to previous years. This, i.e. improvement in exam scores, has also been weakly confirmed by Yourstone et al [15] in their study in operations management course.

In this study more experienced technique to mathematical and programming questions is applied. This technique enables questions to be broken down into simple multiple-choice, true/false or yes/no questions. A quasi-experimental 2-group comparison with one group using student response system and one group of the same course and same tutor receiving standard teaching will be used. A quasi-experiment is selected since students registering in these groups 
independently registered in the courses, and they were not chosen randomly. The independent variable will be the use of student response system during class. The depended variables will be the student in-class engagement, their perception about the technology, and their course grades.

\section{Student response systems}

A student response system is a system employed in a face-to-face setting to poll students and gather immediate feedback in response to inquiries presented by instructors. A non-technical example of a student response system is an instructor asking students to put up their hands to agree or disagree with a given question. A somewhat more sophisticated practice involves the use of colored flashcards, with each color corresponding to a possible answer to a multiple-choice question. The technology subject to that exploratory research is TurningPoint, from Turning Technologies. It offers infrared and radio frequency variations of their student transmitters, and simple USB-based recipients. In addition, its software integrates completely into Microsoft PowerPoint, allowing deliver, assess, and report from within PowerPoint. Some additional effort is required to develop question slides, but since many instructors already use presentation software (particularly instructors in large lecture courses, where the use of student response system is most attractive), the extra effort is minimized.

In order to use student response system the following three activities should be involved: Instruction and questions breaking, response and data display, and data management and study analysis. Therefore, the instructor presents concepts and materials, develops question slides, and then asks for feedback from students. The questions presented by the instructor should spread from simple realistic recall to questions designed specifically to challenge common misconceptions in a given subject. The next section shows that development of effective questions is decisive to the success of teaching with student response systems. The questions are typically in multiple choice or true/false format. Students, in responses, use small remote transmitters which send signals to a recipient that is connected to the instructor's laptop or classroom PC. Software on the instructor's machine instantly compiles and graphs student responses, and these simple graphs might be shown along the presentation slide. Once students find out the distribution of responses (in case that the question is a part of teaching activity) the instructor should obtain the chance to encourage discussion and take students to reconsider the question in groups and to attain an agreement about the best solution. Thus, the instructor often follows the discourse with a second round of questioning, response, and display before wrapping up the presentations of a given concept. This is often shouted out "peer education".

One of the beneficial sides of using a student response system is that students can compare their own answers to the reactions of other students in the course of study, which can encourage a level of metacognition that might not otherwise happen. Student response systems also allow instructors to export and save response data for future analysis and evaluation. Furthermore, the systems also can integrate with learning management systems such Blackboard and Moodle to simplify the assessment process. Of course there are more advantages that are visible such as scoring capability, correction time and covering considerable material very efficiently.

\section{Instruction and questions breaking}

The creations of questions that are defying, adjusted to the appropriate level, and intellectually worthy have been of a great challenge. In that regard, there are several question types such as multiple choices (or alternatives), short essay, numerical response, demographic undertaking, priority ranking and true/false. A well-known multiple choice question may have, in the average, 5 answer preferences. A numeric response question requires participants to rejoin with a numeric value. A short answer question requires participants to respond with an abridged phrase. Only participants using a specific case of SRS, see [11], [14], may respond to an essay question as it involves software that depends on the natural language processing techniques of information extraction and draws an analogy form based on the verb and subject of model results with each student response. A demographic assignment question which may have about 5 answer choices can be used to assign participants to teams for competitions. Priority ranking questions may have up to 10 answer choices such as items are ranked by the participants and then graded based on response weights. A true or false question requires participants to respond with 1 (or a) for true or 2 (or b) for false. It is worth noting that, we might construct a test using paired (or multi) multiple-choice questions along the same topic with a scoring rule which awarded a bonus if they beat both (or all) questions right. There are various approaches which the tutors can use to analyze each of these question types such as classical test theory, factor analysis, cluster analysis, item response theory, and model analysis, see Agresti and Finlay [1]. These approaches are valuable tools for studies on large-scale measurement using multiple-choice assessments. In this survey, we will use the classical test theory.

In principle, the nature of mathematical and logical statements (used in programming courses) gives the impression that they would lean to the multiple choice format which is considerably conventional to all students. In contrast to some other disciplines of knowledge, many questions in mathematics and logic always have a single and an objectively correct answer, with all other answers being agreed upon as being wrong. One could say that the multiple choice questions might promote the idea that the answer to any mathematics and programming questions is more important than the process utilized to get at that result. In contrary, the process is far more significant than the answer, particularly if the answer is to a mannered question. Knowing how frequently a student gets an answer mathematics and 
programming questions - even with incorrect one - would give the tutor a detailed picture of how the student would be to handle comparable (or more sophisticated) questions in the future. This also allows for a more valuable feedback in the grading process than simply reporting whether a given query was answered correctly or incorrectly, by identifying specific strengths and weaknesses in a student's reasoning.

Now, we are in a spot to illustrate our technique with a first level calculus course, though the point is surely applicable to more advanced mathematics subjects. Suppose this session is meant to teach students how to solve single variable indefinite integrals. Consider the traditional question "Evaluate the indefinite integral $\int x^{2} \ln x d x$ ". There are of course several traps that the student encounters when actually attempting to solve such integration; a common one is commencing with the starting method of integration. One could focus on integration techniques such as integration by parts. At that point, the student should well realize the cause of the troubles of choosing different integration method such as integration by substitution. This may take days or even weeks after first learning the suitable method; without reinforcement, the same error may then recur later in the course, or in subsequent courses. This subjective question is with different answers having different strengths and weaknesses; there is no single "correct" or even "best" answer here. This is how a self-administered multiple choice quiz (in particular, an online quiz) can help, with questions as presented in Table 1. Such questions can be addressed with quick feedback, whether one has any misunderstanding on this specific point, without needing the live intervention of the tutor.

One could come up with some useful multiple choice questions for self-examination for other purposes, even for rather courses. For instance, consider the question testing "how to make loops" in the object programming language Java. Multiple choice questions seem most effective here for reinforcing the precise definition of loops and how students determine the appropriate type loops. Loops are what you use to perform similar processes over and over. Likewise, it is possible to suppose that "while loop" can be used for all of your looping needs. Nevertheless, it is a standard that if you know in advance how many times you need to get through your loop, you should use a "for loop". There is one other looping construct available in Java, which is "do... while loop", in which case, students can employ it. This is standardized to the indefinite integral problem mentioned above.

Table 1: Questions Testing How to Solve the Indefinite Integral $\int x^{2} \ln x d x$

$\mathrm{Q}_{1}$. Which of the following integration method you will first use to evaluate $\int \ln x d x$ :

a) By parts.

b) By substitution.

c) By partial fractions.

d) By trigonometric substitution.

e) By rationalization.

$\mathrm{Q}_{2}$. Which of the following technique you will first use to evaluate $\int \ln x d x$ :

a) Integration by parts, differentiating $\ln x$ and integrating 1 .

b) Integration by parts, differentiating 1 and integrating $\ln x$.

c) Integration by substitution, setting $y=x$.

d) Integration by substitution, setting $y=\ln (x)$.

e) Integration by substitution, setting $y=e^{x}$.

\section{$\mathrm{Q}_{3} \cdot \int \ln x d x=$}

a) $\quad x \ln x+x+c$.

b) $\quad x \ln x-x+c$.

c) $\quad(x+1) \ln x+c$.

d) $\quad(x+1) \ln x+x+c$.

e) $\quad(x-1) \ln x-x+c$.

$\mathrm{Q}_{4}$. Which of the following technique you will first use to evaluate $\int x^{2} \ln x d x$ :
a) Integration by parts, differentiating $\ln x$ and integrating $x^{2}$.
b) Integration by parts, differentiating $x^{2}$ and integrating $\ln x$.
c) Integration by substitution, setting $y=x$.
d) Integration by substitution, setting $y=\ln x$.
e) Integration by substitution, setting $y=x^{2}$. 
Q5. $\int x^{2} \ln x d x=$

a) $\quad \frac{1}{3} x^{3} \ln x-\frac{1}{3} x^{3}+C$.

b) $\quad \frac{1}{9} x^{3} \ln x+\frac{1}{3} x^{3}+C$.

c) $\quad \frac{1}{3} x^{3} \ln x+\frac{1}{9} x^{3}+C$.

d) $\quad \frac{1}{9} x^{3} \ln x-\frac{1}{3} x^{3}+C$.

e) $\quad \frac{1}{3} x^{3} \ln x-\frac{1}{9} x^{3}+C$

\section{Response and data display}

Participants can interact through response devices (response cards) that communicate through a receiver or the internet with web enabled devices (response ware). In that respect are different systems (or schemes) employed by the transmitters to send signals to the recipients (or some time receive signals from the recipient) such as infrared, radio frequency, and Wi-Fi systems. Each of these systems has preferences and drawbacks, and deciding which system is best depends heavily upon the needs and priorities for a given context. We need a receiver which only accepts the first response sent by each participant and, hence, a correct answer indicator allows participants to see the correct answer or answers after polling is closed. This indicator appears during a presentation when the slide is advanced after displaying the chart.

Infrared (IR) systems essentially employ the same term-of-sight technology that is utilized in domestic television remote control devices. They are ranking below other equipments in terms of cost. Moreover, there are no issues from classroom to classroom as signals do not pass through the walls of the hall. They minimize the expectation that students could engage in each other's transmitter when responses are exercised for attendance. However, the clickers have to be directed straight to the receivers in order to work and thus have peak seeing in the classroom. So far, most IR systems put forward only one-way communication, which does not authorize for confirmation when the student's answer has been received. They also require the placement of receivers in line-of-sight of students, which often stands for permanent or semi-permanent installation. Each receiver can only take less than 100 transmitters (depending on industrialist), so numerous receivers are needful for considerable sections. Over and above, clicker administration and management can be expensive and in very large classes, signal reception can be uncertain and have a shorter range.

In radio frequency (RF) systems, the receiver should not be in line-of-sight of students, facilitating hardware solutions and making signal reception is more reliable and delivers a longer reach. Radio frequency systems also allow two-way communications, so response pad can confirm when the student's response has been obtained. Nevertheless, low visibility might make it more agreeable for students to deceive the system by fetching in each other's transmitter when responses are applied for being present. The RF technology clickers as well as its administration and management are more expensive than IR. There is also a series disadvantage which is the higher likelihood of interference issues as RF clickers can operate on the same frequencies as Wi-Fi and other RF devices.

Wi-Fi systems employ a web-based interface for student interaction. They allow for text entry and open-ended responses. Students can employ a wide variety of Wi-Fi devices and also the existing campus wireless infrastructure. However, it requires students to have a Wi-Fi computing device or smart phones. There are also fewer choices actually available in the market place.

Different types of correct answer indicators such as rectangle, checkmark smiley, star, arrow and custom can be used. A chart displays the results of participant responses. The default chart type and display options, such as colors, labels and value, can be defined in the preferences. Also the live results monitor provides real-time information about a class test. The monitor displays which questions each participant has answered and whether they responded correctly or incorrectly. There is also an option to view the overall progress for the entire test. In addition, there are several types of session reports such as results by question, results by participant, results retail, results by demographic, comparative results and session log report.

All session files are associated with a participant list that resembles a grade book. A participant list is a list of participant names, user IDs, device IDs and other custom categories. The use of a participant list allows for detailed reporting on each participant from a session. Exported participant information from integration can also be imported as a participant list. The results manager overview all of the sessions associated with a participant information, number of columns, number of participants, maximum points and average score. Moreover, LMS integrations allow for the sharing of data between SRS and the LMS. Sessions can be exported to the LMS grade book directly from SRS. An LMS Integration must be configured on an LMS server. Also, I would prefer to update participant list information from the LMS integration and to confirm with IT department that the integration is properly setup before attempting to use the integration in SRS. 


\section{Data management and study analysis}

The data (result) manager of any response system should provide tutors by different types of session reports. By selecting the data option tutor can view results by question, results by participant, all results detail, results by demographic, comparative results and session log report and performance scale. In summation, a performance scale can be set to display a text or numeric value equal to how the participant has performed across all sessions. Sessions can also have performance points, attendance points and grades that can be manually edited. Moreover, a session can be turned out from the overall grade and average calculations. As a close, any sessions associated with a participant list are automatically generated into the results manager.

For example, the result of question report should include the difficulty index and discrimination index. The difficulty index is a measure of how difficult the question is to correctly answer. It shows the percentage of participants that answered the question correctly based on the entire group of active participants. The difficulty index is calculated using the formula $P=N_{1} / N$, where $N_{1}$ the number of is correct responses and $N$ is the total number of students taking the test. The discrimination index for a particular question which is based on the point biserial correlation coefficient measures the correlation between the correct answer on a question and the total test scores of the active participants. The discrimination index can then be computed with the formula

$$
D=\frac{\overline{X_{1}}-\overline{X_{0}}}{\sigma_{x}} \sqrt{\frac{N_{1} N_{0}}{\left(N_{1}+N_{0}\right)\left(N_{1}+N_{0}-1\right)}} .
$$

Here $\overline{X_{1}}$ is the mean total score for those who correctly answered the question, $\overline{X_{0}}$ is the mean total score for those who incorrectly answered the question, $\sigma_{x}$ is the standard deviation of total scores, $N_{1}$ is the total number of participants that answered the question correctly and $N_{0}$ is the total number of participants that answered the question incorrectly. Practically, see Ding and Beichner [3], question difficulty values ranging from 0.3 to 0.9 are acceptable while a commonly adopted standard for a satisfactory discriminatory index is $D \geq 0.3$.

A quasi-experimental approach that has been employed in this study is often the best possible to compare outcomes, e.g. if one asks different classes taking the same form for participation, one division might be taught by using SRS and the other one using traditional instruction. Furthermore, it might be applied to compare outcomes for one group of individuals before and after the groups' interest in using SRS technology. For example, the knowledge, behaviors and grades of students in the fall are compared with the same measures in the spring. However, Quasi experimental studies can inform discussions of cause and effect, but, unlike true experiments, they cannot definitively prove this connection. In this exploratory research, I studied the utilization of an SRS in two different courses, with each class capacity of 30 students, taught to Information Technology and Computing Program (ITC), AOU, and its power to actively engage students in an otherwise traditional lecture setting. Overall statistics show that the usage of such technology adds value to the class and enhances participants' attention, enjoyment, and overall learning. Log files indicate that on average around $98 \%$ of the students in the classroom attempt to answer questions during a lecture using the arrangement which is higher than what we expect in traditional classroom settings. Data for this research presents a first-use of this technology by the author, and the resolutions suggest that equipment reliability and/or instructor competence significantly influences the quality of students' experience and may be biasing these initial results. This exploratory survey also offered valuable insight into student perceptions of interactive technologies in general, and how those perceptions are affected by system responsiveness and accuracy.

\section{Conclusion}

There are various and interesting techniques - many of which appear to be unexplored at present - in which some wellformulated and self-administered online questions can efficiently assess one's strengths and weaknesses in a given mathematical and computational work. Of course, having one-on-one interaction with a lecturer would be a greatly preferable way to gain this kind of instant feedback; merely this is impractical for larger classes. Moreover, it is substantial that a certain degree of maturity and discipline is demanded along the student's side to actually benefit from these self-assessments since they might not be directly contributing to the student's grade in the course of study.

The system that breaks mathematics and programming problems into steps can be assessing all the skills involved in problem solving. Then we let computers do marking, relieving human markers of more or less of the overwork of marking and freeing up time for them to assess what they and only they can assess with originality. I can pronounce that such system makes for a very useful supporting role in self-examination for different forms, especially with regards to foundational material (e.g. definitions or basic principles of deliberation). The point is certainly applicable to more advanced topics in such courses. In summary, the assessment results have indicated that the integration of SRS in the AOU curriculum has been successful in its initial stage of evolution; I think that the wide extent to which SRS can be an effective active-learning tool. My next aim is to utilize such system as a refilling of the tutor marked assessment (TMA). 


\section{References}

[1] Agresti, A. and Finlay, B., "Statistical Methods for the Social Sciences", 4th ed., Prentice-Hall Upper Saddle River NJ, 2009.

[2] Chickering, A.W. and Gamson, Z.F., "Seven Principles for Good Practice in Undergraduate education", Biochemical Education, vol. 17, no.3, pp. 140-141, 1989. http://dx.doi.org/10.1016/0307-4412(89)90094-0.

[3] Ding, L. and Beichner, R., "Approaches to Data Analysis of Multiple-Choice Questions", Physical Review Special Topics - Physical Education Research 5 020103, pp. 1-17, 2009

[4] Freeman, J. and Dobbie, A., "Use of an Audience Response System to Augment Interactive Learning", Family Medicine, vol. 37, no.1, pp. 12-14, 2005.

[5] Guthrie, R.W. and Carlin, A., "Waking the Dead: Using Interactive Technology to Engage Passive Listeners in the Classroom", in Proceedings of the Tenth Americas Conference on Information Systems New York NY, pp. 1-8, 2004.

[6] Hall, R.H., Collier, H.L., Thomas, M.L. and Hilgers, M.G., "A Student Response System for Increasing Engagement, Motivation, and Learning in High Enrollment Lectures", in Proceedings of the Americas Conference on Information Systems, pp. 621-626, 2005.

[7] Miller, T., "Formative Computer-Based Assessment in Higher Education: The effectiveness of Feedback in Supporting Student Learning", Assessment \& Evaluation in Higher Education, vol. 34, no.2, pp. 181-192, 2008. http://dx.doi.org/10.1080/02602930801956075.

[8] Morling, B., McAuliffe, M., Cohen, L. and DiLorenzo, T.M., "Efficacy of Personal Response Systems ("Clickers") in Large, Introductory Psychology Classes”, Teaching of Psychology, vol. 35, no.1, pp. 45-50, 2008. http://dx.doi.org/10.1080/00986280701818516.

[9] Sayed, M., "Blended Learning Environment: The Effectiveness in Developing Concepts and Thinking Skills", Journal of Education and Practice, vol. 4, no. 25, pp. 12-17, 2013.

[10] Sayed, M. and Baker, F., "Blended Learning Barriers: An Investigation, Exposition and Solutions", Journal of Education and Practice, vol. 5, no. 6, pp. 81-85, 2014.

[11] Simpson, V. and Oliver, M., "Electronic Voting Systems for Lectures then and Now: A comparison of Research and Practice", Australasian Journal of Educational Technology, vol. 23, no. 2, pp. 187-208, 2007.

[12] Stein, P.S., Challman, S.D. and Brueckner, J.K., "Using Audience Response Technology for Pretest Reviews in an Undergraduate Nursing Course", Journal of Nursing Education, vol. 45, no. 11, pp. 469-473, 2006.

[13] Torbeck, L., "Enhancing Programme Evaluation Using the Audience Response System", Medical Education, vol. 41, no. 11, pp. 1088-1089, 2007. http://dx.doi.org/10.1111/j.1365-2923.2007.02864.x.

[14] Vojak, C., Kline, S., Cope, B., McCarthey, S. and Kalantzis, M., "New Spaces and Old Places: An Analysis of Writing Assessment Software", Computers \& Composition, vol. 28, pp. 97-111, 2011. http://dx.doi.org/10.1016/j.compcom.2011.04.004.

[15] Yourstone, S.A., Kraye, H.S. and Albaum, G., "Classroom Questioning with Immediate Electronic Response: Do Clickers Improve Learning?", Decision Sciences Journal of Innovative Education, vol. 6, no. 1, pp. 75-88, 2008. http://dx.doi.org/10.1111/j.15404609.2007.00166.x. 